\title{
Planktonic Cyanobacteria forming blooms in reservoirs of northeastern Brazil
}

\author{
Nísia K. C. Aragão-Tavares ${ }^{1}$, Ariadne do N. Moura ${ }^{1} \&$ Maria do Carmo Bittencourt-Oliveira ${ }^{2}$ \\ ${ }^{1}$ Universidade Federal Rural de Pernambuco, Departamento de Biologia, Área de Botânica, Rua Dom Manoel de Medeiros, s/n, Dois Irmãos, CEP 52171-900, Recife-PE, Brasil. \\ E-mail: nisiakarine@yahoo.com.br; ariadne_moura@hotmail.com \\ ${ }^{2}$ Universidade de São Paulo, Escola Superior de Agricultura Luiz de Queiroz, Departamento de Ciências Biológicas, Av. Pádua Dias, 11, Universitário, CEP 13418-900, Piracicaba-SP, \\ Brasil. Caixa Postal 9. E-mail: mbitt@usp.br
}

\section{ABSTRACT}

This paper contributes to the inventory of cyanobacteria in freshwater environments in Northeastern Brazil. Forty-two samples were collected from 19 reservoirs between February 2009 and January 2010. Twenty-three species of cyanobacteria distributed among the orders Chroococcales (10), Oscillatoriales (8) and Nostocales (5) were identified, 12 of which constitute new records for the state of Pernambuco. The greatest degrees of species richness were recorded in three ecosystems (Alagoinha, Carpina and Ingazeira reservoirs). Microcystis was the most representative genus, with the greatest number of species (Microcystis novacekii (Komárek) Compère, Microcystis panniformis J. Komárek, J. Komárková-Legnerová, C.L. Sant'Anna, M.T.P. Azevedo \& P.A.C, Senna, Microcystis protocystis W.B. Crow and Microcystis sp.). Cylindrospermopsis raciborskii (Woloszynska) Seenaya and Subba Raju (straight morphotype) had the greatest distribution (considered very frequent) and was present in 16 reservoirs (84.25\%). Only four of the 42 samples did not have cyanobacteria.

Key words: cyanophyta, phytogeographic distribution, species richness, taxonomy

\section{Cianobactérias planctônicas formadoras de florações em reservatórios do nordeste do Brasil}

\section{RESUMO}

Este trabalho contribui para o inventário das cianobactérias de ambientes de água doce do Nordeste do Brasil. 42 amostras foram coletadas em 19 reservatórios, entre fevereiro de 2009 e janeiro de 2010. Foram identificadas 23 espécies de cianobactérias distribuídas nas ordens Chroococcales (10), Oscillatoriales (8) e Nostocales (5), dentre as quais 12 se constituem em novas citações para o estado de Pernambuco. A maior riqueza de espécies foi registrada em três ecossistemas (Reservatórios de Alagoinha, Carpina e Ingazeira). Microcystis foi o gênero mais representativo com maior número de espécies (Microcystis novacekii (Komárek) Compère, Microcystis panniformis J. Komárek, J. Komárková-Legnerová, C. L. Sant'Anna, M. T. P. Azevedo \& P. A. C, Senna, Microcystis protocystis W. B. Crow and Microcystis sp.). Cylindrospermopsis raciborskii (Woloszynska) Seenaya e Subba Raju (morfotipo reto) apresentaram maior distribuição (considerada muito frequente), presentes em 16 reservatórios (84.25\%). Apenas quatro das 42 amostras não apresentaram cianobactérias.

Palavras-chave: cyanophyta, distribuição fitogeográfica, riqueza de espécies, taxonomia 


\section{Introduction}

Cyanobacteria are cosmopolitan organisms considered to be the first photosynthesizers with chlorophyll a and primary producers to release oxygen into the atmosphere (Chorus \& Bartram, 1999). These organisms exhibit broad ecological tolerance, which contributes to their competitive success. One of the striking characteristics of this group is the ability to grow in the most varied environments - from hot springs to cold oceans as well as in terrestrial environments (Domitrovic \& Forastier, 2005).

However, freshwater ecosystems are the most suitable environments for the development of cyanobacteria, especially in tropical regions, which have the most favorable conditions for cyanobacterial blooms - a phenomenon characterized by the intensive growth of these microorganisms in water (Chorus \& Bartram, 1999). As well as increases in nutrients, changes in temperature and mixing regime are important factors related to these blooms (Paerl \& Huisman, 2008).

Cyanobacteria possess a range of unique and highlyadaptable eco-physiological traits (Litchman et al., 2010). These traits, which can be specific at the genus level, such as: 1) the ability to grow in warmer temperatures; 2) buoyancy, due to gas vesicle production; 3) high affinity for, and ability to store, phosphorus; 4) nitrogen-fixation; 5) akinete production and associated life history characteristics; and 6) light capture at low intensities (Carey et al., 2012). Reservoirs in Northeastern Brazil offer these conditions and therefore favor the occurrence of cyanobacterial populations (Bittencourt-Oliveira \& Molica, 2003; Chellapa et al., 2008; Dantas et al., 2008, 2012; Moura et al., 2007, 2010, 2011).

The extensive morphological plasticity and overlapping measures in species of cyanobacteria have caused problems in the correct identification of taxa, and therefore, according to Bittencourt-Oliveira et al. (2001), this can lead to a false understanding of the toxic potential of various species. Due to these problems, there are few works that aim as the primary focus aspects of taxonomic group, and thus, only about $5-10 \%$ of this diversity is known (Komárek, 2003).

In Pernambuco, studies on cyanobacteria began with Carvalho-De-La-Mora (1986) and were intensified after the "syndrome of Hemodialysis". Jochimsen et al. (1998) reported the occurrence of microcystin in the hemodialysis equipment of the Institute of kidney Diseases in Caruaru City (PernambucoBrazil) the place of contamination and death of humans by cyanotoxins. Then, Domingos et al. (1999) confirmed the production of microcystin by picoplankton cyanobacteria isolated from material collected in two reservoirs in the state of Pernambuco (Tabocas and Sr. José Maria), used to supply the city of Caruaru, suggesting that these organisms may have contributed to human poisoning in hemodialysis clinic of the municipality.

Then, several studies with distinct approaches (ecology, taxonomy, seasonal and spatial distribution) were carried out with cyanobacteria in many reservoirs used for public supply in the state of Pernambuco, (Bouvy et al., 2000, 2001, 2003; Huszar et al., 2000; Aragão et al., 2007; Dantas et al., 2008, 2011; Lira et al., 2010; Moura et al., 2010, 2011; Bittencourt-Oliveira et al., 2011). Considering the frequent blooms of cyanobacteria and the problems posed to aquatic ecosystems, the present study aimed to contribute to the knowledge of the diversity of these algae in reservoirs in Northeast of Brazil, since, in most cases, the blooms are formed by species potentially toxic, causing a risk to public health.

\section{Material and Methods}

\section{Study Area and Sampling}

Forty-two samples were taken from 19 reservoirs located in different phytogeographic regions in the state of Pernambuco (Figure 1) (Table 1), which are characterized by high temperatures and dry periods, especially between October and March (summer); the winter (April to September) is featured by greater precipitation and milder temperatures. These ecosystems are used mainly for the public water supply and fishing activities of about three million inhabitants (ANA, 2012).

Sampling was carried out at a single point at the subsurface near the bank between February 2009 and January 2010. The taxonomic study was performed based on semi-permanent slides with samples of living matter analyzed immediately after collection and complemented with samples preserved in formalin and Transeau solution. Analyses were performed with the aid of a ZEISS optical microscope (Jenaval model) with a measurement ocular (magnification: 400X and 1000X).

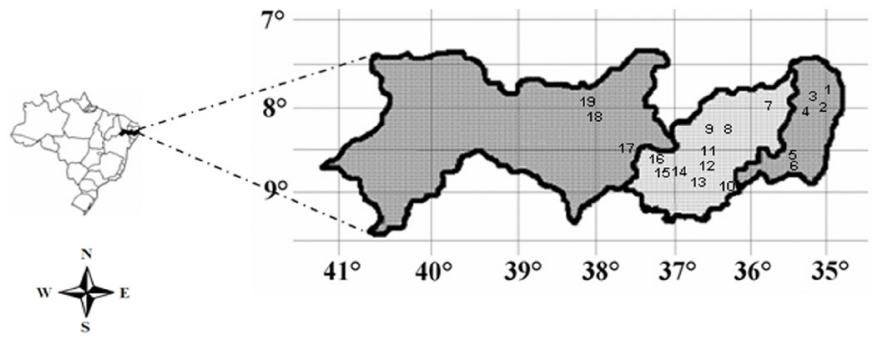

Figure 1. Map of location of the reservoirs in the state of Pernambuco. 1Botafogo, 2- Duas Unas, 3- Tapacurá, 4- Carpina, 5- Pastora, 6- Santo Antônio dos Palmares, 7- Jucazinho, 8- Bitury, 9- Ipojuca, 10- Mundaú, 11- Alagoinha, 12- Venturosa, 13- Ingazeira, 14- Pedra, 15- Arcoverde, 16- Buíque, 17- Poço da Cruz, 18- Jazigo, 19- Saco I

Morphological taxonomic characteristics of the organisms were analyzed, such as shape and dimensions of the heterocyst, akinetes, trichomes and cells as well as the presence of aerotopes and coloration. Identification was carried out to the smallest possible taxonomic level using the specialized literature (Komárek \& Anagnostidis, 1986; 2005; Anagnostidis \& Komárek, 1988; Cronberg \& Annadotter, 2006; Komárek \& Zapomělová, 2007).

After analysis and taxonomic photomicrographs, samples preserved with formalin, were deposited in the Herbarium Professor Vasconcelos Sobrinho, Federal Rural University of Pernambuco (PEURF 50435 to 50476).

Frequency of occurrence was calculated based on the system proposed by Mateucci \& Colma (1982), considering the following categories: $>70 \%=$ very frequent $(\mathrm{VF}) ; \leq 70 \%$ and $>40 \%=$ frequent $(\mathrm{F}) ; \leq 40 \%$ and $>10 \%=$ occasional $(\mathrm{O})$; and $\leq 10 \%=$ sporadic or rare $(\mathrm{S})$. 
Table 1. Location of the reservoirs, sampling dates, phytogeographical region and coordinates

\begin{tabular}{|c|c|c|c|}
\hline Reservoirs (City) & Sampling dates & Phytogeographical region & Coordinates \\
\hline Alagoinha (Alagoinha) & $04 / 2009,10 / 2009$ & Agreste & $8^{\circ} 27^{\prime} 31.9^{\prime \prime} \mathrm{S}, 6^{\circ} 46^{\prime} 33.5^{\prime \prime} \mathrm{W}$ \\
\hline Arcoverde (Buíque) & $05 / 2009,11 / 2009$ & Agreste & $8^{\circ} 33^{\prime} 32.5^{\prime \prime}$ S, 6 $6^{\circ} 59^{\prime} 07.5^{\prime \prime} \mathrm{W}$ \\
\hline Bitury (Belo Jardim) & $02 / 2009,04 / 2009$ & Agreste & $08^{\circ} 18^{\prime} 35^{\prime \prime} \mathrm{S}, 36^{\circ} 25^{\prime} 36^{\prime \prime} \mathrm{W}$ \\
\hline Botafogo (Igarassu) & $05 / 2009,10 / 2009$ & Litorânea & $7^{\circ} 50^{\prime} 11.8^{\prime \prime} \mathrm{S}, 35^{\circ} 02^{\prime} 0.8^{\prime \prime} \mathrm{W}$ \\
\hline Buíque (Buique) & $06 / 2009,11 / 2009$ & Agreste & $8^{\circ} 37^{\prime} 52.7^{\prime \prime} \mathrm{S}, 7^{\circ} 07^{\prime} 53.5^{\prime \prime} \mathrm{W}$ \\
\hline Carpina (Lagoa do carro) & $04 / 2009,10 / 2009$ & Zona da Mata & $7^{\circ} 53^{\prime} 03.8^{\prime \prime} \mathrm{S}, 5^{\circ} 20^{\prime} 37.8^{\prime \prime} \mathrm{W}$ \\
\hline Duas Unas (Jaboatão dos Guararapes) & $05 / 2009,10 / 2009$ & Zona da Mata & $8^{\circ} 05^{\prime} 02^{\prime \prime}$ S, $35^{\circ} 30.6^{\prime \prime} \mathrm{W}$ \\
\hline Ingazeira (Venturosa) & $04 / 2009,10 / 2009$ & Agreste & $8^{\circ} 36^{\prime} 41.2^{\prime \prime} \mathrm{S}, 6^{\circ} 54^{\prime} 23.7^{\prime \prime} \mathrm{W}$ \\
\hline Ipojuca (Belo Jardim) & $04 / 2009,11 / 2009$ & Agreste & $8^{\circ} 20^{\prime} 43.7^{\prime \prime} \mathrm{S}, 36^{\circ} 22^{\prime} 31.5^{\prime \prime} \mathrm{W}$ \\
\hline Jazigo (Serra Talhada) & $05 / 2009,01 / 2010$ & Sertão & $8^{\circ} 00^{\prime} 08.2^{\prime \prime}$ S, $38^{\circ} 12^{\prime} 38.5^{\prime \prime} \mathrm{W}$ \\
\hline Jucazinho (Surubim) & $03 / 2009,10 / 2009$ & Agreste & $7^{\circ} 59^{\prime} 03^{\prime \prime}$ S, $35^{\circ} 48^{\prime} 36.7^{\prime \prime} \mathrm{W}$ \\
\hline Mundaú (Garanhuns) & $03 / 2009,11 / 2009$ & Agreste & $8^{\circ} 56^{\prime} 42.8^{\prime \prime}$ S, 36²9'27.4” W \\
\hline Pastora (Palmares) & $06 / 2009$ & Zona da Mata & $08^{\circ} 41^{\prime} 28.5^{\prime \prime} \mathrm{S}, 5^{\circ} 36^{\prime} 53.8^{\prime \prime} \mathrm{W}$ \\
\hline Pedra (Pedra) & $06 / 2009,11 / 2009$ & Agreste & $8^{\circ} 29^{\prime} 37^{\prime \prime} \mathrm{S}, 36^{\circ} 56^{\prime} 40^{\prime \prime} \mathrm{W}$ \\
\hline Poço da Cruz (Ibimirim) & $05 / 2009,01 / 2010$ & Sertão & $8^{\circ} 30^{\prime} 31.5^{\prime \prime}$ S, $37^{\circ} 42^{\prime} 17.9^{\prime \prime} \mathrm{W}$ \\
\hline Saco I (Serra Talhada) & $05 / 2009,01 / 2010$ & Sertão & $7^{\circ} 56^{\prime} 49.3^{\prime \prime}$ S, 38¹7'13.1" W \\
\hline Santo Antônio dos Palmares (Palmares) & $06 / 2009$ & Zona da Mata & $08^{\circ} 41^{\prime} 35.7^{\prime \prime} \mathrm{S}, 5^{\circ} 39^{\prime} 24.6^{\prime \prime} \mathrm{W}$ \\
\hline Tapacurá (São Lourenço da Mata) & $03 / 2009,10 / 2009$ & Litorânea & $8^{\circ} 02^{\prime} 31.9^{\prime \prime} \mathrm{S}, 35^{\circ} 11^{\prime} 46.5^{\prime \prime} \mathrm{W}$ \\
\hline Venturosa (Venturosa) & $04 / 2009,10 / 2009$ & Agreste & 8'34'43.6” S, 36॰52'47.3” W \\
\hline
\end{tabular}

\section{Results and Discussion}

Cyanobacteria were recorded in 38 of the 42 samples analyzed. Twenty-three species were identified in the 19 ecosystems, distributed among the orders Oscillatoriales, $(10$ spp.), Chroococcales (8 spp.) and Nostocales (5 spp.) (Table 2 ). The greatest degrees of species richness were recorded in the Alagoinha, Carpina and Ingazeira reservoirs (10 spp.). Cylindrospermopsis raciborskii (Woloszynska) Seenayya et Subba Raju and Sphaerospermopsis aphanizomenoides (Forti) Zapomelová, Jezberová, Hrouzek, Hisem, Reháková \& Komárková were present in all samples from these reservoirs.
The total number of species identified was relatively smaller than that recorded in previous studies with emphasis at cyanobacteria community at Northeastern of Brazil (Aragão et al., 2007; Costa et al., 2009; Dantas et al., 2011; Lira et al., 2011) who recorded greater cyanobacterial richness. The lower number of taxa in the present study is likely due to the occurrence of blooms dominated by one or more species of cyanobacteria in the majority of reservoirs studied. The genus Microcystis was the most representative, with four species ( $M$. novacekii (Komárek) Compère, $M$. panniformis J. Komárek, J. Komárková-Legnerová, C.L. Sant'Anna, M.T.P. Azevedo \& P.A.C, Senna, M. protocystis W.B. Crow and Microcystis sp.). These same species were

Table 2. Taxa of Cyanobacteria and frequence of occurrence (F.O. \%) in 19 reservoirs of semiarid region of state of Pernambuco. Reservoir: 1-Alagoinha, 2-Arcoverde, 3-Bitury, 4-Botafogo, 5-Buíque, 6-Carpina, 7-Duas Unas, 8-Ingazeira, 9-Ipojuca, 10-Jazigo, 11-Jucazinho, 12-Mundaú, 13-Pastora, 14-Pedra, 15-Poço da Cruz, 16-Saco I, 17-Santo Antônio dos Palmares, 18-Tapacurá and 19-Venturosa

\begin{tabular}{|c|c|c|c|c|c|c|c|c|c|c|c|c|c|c|c|c|c|c|c|c|}
\hline Taxa/Reservoirs & 1 & 2 & 3 & 4 & 5 & 6 & 7 & 8 & 9 & 10 & 11 & 12 & 13 & 14 & 15 & 16 & 17 & 18 & 19 & F.O. (\%) \\
\hline \multicolumn{21}{|l|}{ Chroococcaceae } \\
\hline Chroococcus obliteratus & - & - & - & - & - & - & - & + & - & - & + & - & - & - & - & - & - & - & - & $10.52(0)$ \\
\hline Chroococcus sp. & + & - & - & - & - & + & - & - & - & - & - & - & - & + & + & - & - & - & + & $26.31(0)$ \\
\hline Merismopedia glauca & - & + & - & - & + & - & - & + & - & - & - & - & + & - & - & + & - & - & - & $26.31(0)$ \\
\hline M. tenuissima & + & + & - & + & - & - & - & + & - & - & - & + & - & + & - & - & - & + & + & $42.10(F)$ \\
\hline \multicolumn{21}{|l|}{ Microcystaceae } \\
\hline Microcystis novacekii & + & - & - & - & - & - & + & - & - & - & - & + & - & + & - & - & - & - & - & $21.05(0)$ \\
\hline M. panniformis & + & + & - & - & - & + & + & - & - & - & - & + & - & + & - & - & - & + & - & $36.84(0)$ \\
\hline M. protocystis & + & - & - & - & - & - & - & + & - & - & - & + & - & - & - & - & - & + & - & $21.05(0)$ \\
\hline Microcystis sp. & + & - & - & + & + & - & + & - & + & - & + & + & - & - & - & - & - & + & - & $42.10(F)$ \\
\hline \multicolumn{21}{|l|}{ Oscillatoriaceae } \\
\hline Lyngbya cf. ceylanica & - & - & - & - & - & - & - & - & + & - & - & - & - & - & - & - & - & - & - & $5.26(\mathrm{~S})$ \\
\hline Oscillatoria princeps & - & + & - & - & - & - & - & - & - & - & - & - & + & - & - & - & - & - & - & $10.52(0)$ \\
\hline Oscillatoria sp. & - & - & - & - & - & + & - & - & - & + & + & - & - & + & - & - & - & + & - & $26.31(0)$ \\
\hline \multicolumn{21}{|l|}{ Phormidiaceae } \\
\hline Planktothrix agardhii & + & + & - & - & - & + & + & + & + & - & + & - & - & - & - & - & + & + & - & $47.36(\mathrm{~F})$ \\
\hline P. isothrix & - & - & - & - & - & + & - & - & + & - & + & - & - & + & - & - & - & - & - & $21.05(0)$ \\
\hline Planktothrix sp. & - & - & - & + & - & - & - & - & - & + & - & - & - & - & + & + & + & - & - & $26.31(0)$ \\
\hline \multicolumn{21}{|l|}{ Pseudanabaenaceae } \\
\hline Geitlerinema amphibium & + & + & + & - & + & + & + & + & + & - & + & + & - & + & + & + & - & + & - & 73.68(VF) \\
\hline Pseudanabaena catenata & + & + & + & + & + & + & - & + & + & - & + & + & - & + & - & - & - & + & - & $63.15(F)$ \\
\hline P. papillaterminata & - & + & - & - & - & - & - & - & - & - & + & - & - & - & + & - & - & + & - & $21.05(0)$ \\
\hline Spirulina major & - & - & - & - & - & + & - & - & - & - & - & - & - & - & - & - & - & - & - & $5.26(\mathrm{~S})$ \\
\hline \multicolumn{21}{|l|}{ Nostocaceae } \\
\hline Cylindrospermopsis raciborskii (Straight) & + & + & + & - & + & + & + & + & + & - & + & + & - & + & + & + & + & + & + & $84.25(\mathrm{VF})$ \\
\hline C. raciborskii (Coiled) & + & - & + & - & + & + & + & + & + & - & + & + & - & + & + & + & - & + & - & $68.42(F)$ \\
\hline Dolichospermum maximum & - & - & - & - & - & - & + & - & - & - & - & - & - & - & - & - & - & - & - & $5.26(\mathrm{~S})$ \\
\hline D. torques-reginae & - & - & - & - & - & + & - & - & - & - & - & - & - & - & - & - & - & + & - & $10.52(0)$ \\
\hline Dolichospermum sp. & + & - & + & - & - & + & - & + & + & - & + & - & - & - & - & - & - & + & - & $36.84(0)$ \\
\hline Sphaerospermopsis aphanizomenoides & + & + & + & + & + & + & + & + & + & - & + & - & - & - & - & + & - & + & - & $63.15(F)$ \\
\hline
\end{tabular}


recorded by Costa et al. (2006) forming blooms in the Armando Ribeiro Gonçalves reservoir in the state of Rio Grande do Norte (Northeastern Brazil).

Ten new taxa are recorded for the state of Pernambuco: Chroococcus obliteratus Richter, Microcystis novacekii, M. protocystis, Lyngbya cf. ceylanica Wille, Planktothrix isothrix (Skuja) Komárek \& Komárková, Pseudanabaena papillaterminata (Kiselev) Kukk, Spirulina major Kützing ex Gomont, Dolichospermum maximum (Cronberg et Komárek) Wacklin, D. torques-reginae (Komárek) Wacklin and Sphaerospermopsis aphanizomenoides. Moreover, the genera Dolichospermum and Sphaerospermopsis were renamed, as previous studies have described these genera as Anabaena and Aphanizomenon, respectively (Wacklin et al., 2009; Zapomělová et al., 2010).

C. raciborskii is one of cyanobacteria bloom-forming species in Brazilian ecosystems and populations with straight and coiled trichomes have been observed in Northeastern Brazil (Bittencourt-Oliveira et al., 2011). The straight morphotype of C. raciborskii and Geitlerinema amphibium (Agardh ex Gomont) Anagnostidis were very frequent species with $84.25 \%$ and $73.68 \%$ of occurrence, followed by the coiled morphotype of C. raciborskii, Merismopedia tenuissima Lemmermann, Microcystis sp., Planktothrix agardhii (Gomont) Anagnostidis \& Komárek, Pseudanabaena catenata Lauterborn and S. aphanizomenoides, which were considered
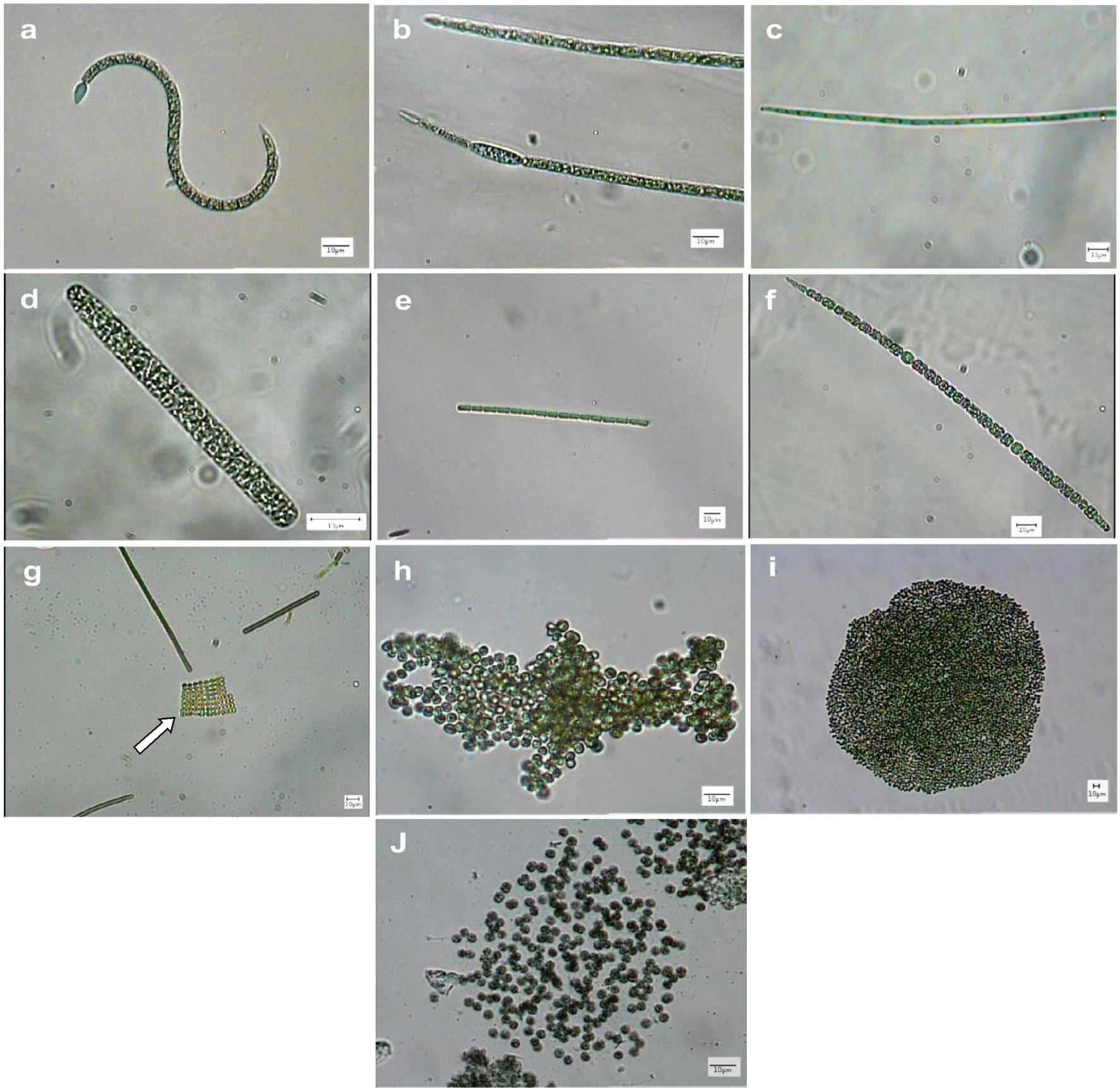

Figure 2. Planktonic Cyanobacteria recorded in reservoirs in the state of Pernambuco. a. Cylindrospermopsis raciborskii coiled morphotype, b. C. raciborskii straight morphotype, c. Geitlerinema amphibium, d. Planktothrix agardhii, e. Pseudanabaena catenata, f. Sphaerospermopsis aphanizomenoides, g. Merismopedia tenuissima, h. Microcystis sp., i. Microcystis panniformis, j. Microcystis protocystis. Scale bar= 10 $\mu m$ 
frequent. D. maximum, Lyngbya cf. ceylanica and S. major, each with a single record $(5.26 \%)$ were considered sporadic species (Table 2).

Among the organisms identified, C. raciborskii, $G$. amphibium, P. agardhii, P. catenata, S. aphanizomenoides, $M$. tenuissima and Microcystis sp. had the broadest distribution, occurring in approximately $42 \%$ to $84 \%$ of the reservoirs (Figure $2 \mathrm{a}-\mathrm{h})$. Some studies have reported the frequent occurrence of C. raciborskii in other Brazilian ecosystems (Bouvy et al., 2000, 2001; Bittencourt-Oliveira \& Molica, 2003; Costa et al., 2006; Aragão et al., 2007; Moura et al., 2007; Panosso et al., 2007; Chellappa et al., 2008; Bittencourt-Oliveira et al., 2011; Dantas et al., 2011; Lira et al., 2011; Moura et al., 2011). The frequency of this species in semiarid ecosystems occurred, in general, when the ecosystem is stratified with a high degree of intermittence in availability of nutrients (Bormans et al., 2005; Dantas et al., 2011).

The frequency, persistence and high density or biomass of one or multispecies of cyanobacteria in drinking reservoir are a greatest problem from the fact of some species, like: $C$. raciborskii, G. amphibium, Microcystis spp., P. agardhii and S. aphanizomenoides are potentially toxic (Figure 2a-b, d, f, $\mathrm{h}-\mathrm{j})$.

\section{Conclusions}

Cyanobacteria were present in all the reservoirs studied, and among the 23 identified species, $C$. raciborskii was the only one considered very common, predominating in most environments. The genus Microcystis was the most representative, with four species (M. novacekii, M. panniformis, M. protocystis and Microcystis sp.). Ten new taxa were recorded for the first time for the state of Pernambuco.

\section{Literature Cited}

Agência Nacional das Águas - ANA. Atlas de Abastecimento Urbano de Água. http://atlas.ana.gov.br/Atlas/forms/ analise/Geral.aspx?est=17. 28 Set. 2012.

Anagnostidis, K.; Komárek, J. Modern approach to the classification system of Cyanophytes, 3: Oscillatoriales. Algological Studies/Archiv für Hydrobiologie, Supplement Volumes, n.50/53, p.327-472, 1988. <http://www. schweizerbart.de/papers/algol_stud/detail/50-53/65276>. 19 Nov. 2012.

Aragão, N. K. C. V.; Gomes, C. T. S.; Lira, G. A. S. T.; Andrade, C. M. Estudo da comunidade fitoplanctônica no reservatório do Carpina-PE, com ênfase em Cyanobacteria. Revista do Instituto Adolfo Lutz, v.66, n.3, p.240-248, 2007. <http://biblioteca.ial.sp.gov.br/ index.php?option $=$ com_remository\&Itemid $=27 \&$ func $=$ fil einfo\&id=359>. 19 Nov. 2012.

Bittencourt-Oliveira, M. C.; Molica, R. Cianobactérias Invasoras. Revista Biotecnologia Ciências e Desenvolvimento, v.30, p.82-90, 2003. <http://www. biotecnologia.com.br/revista/bio30/cianobacteria.pdf $>.19$ Nov. 2012.
Bittencourt-Oliveira, M. C.; Moura, A. N.; Hereman, T. C.; Dantas, E. W. Increase in straight and coiled Cylindrospermopsis raciborskii (Cyanobacteria) populations under conditions of thermal de-stratification in a shallow tropical reservoir. Journal of Water Resource and Protection, v.3, n.4, p.245-252, 2011. <http://dx.doi. org/10.4236/jwarp.2011.34031>.

Bittencourt-Oliveira, M. C.; Oliveira, M. C.; Bolch, C. J. S. Genetic variability of Brazilian strains of the Microcystis aeruginosa complex (Cyanophyceae/Cyanobacteria) using the nucleotide sequence of the intergenic spacer and flanking regions from $c p c \mathrm{BA}$-phycocyanin operon. Journal of Phycology, v.37, n.5, p.810-818, 2001. <http://dx.doi.or $\mathrm{g} / 10.1046 / \mathrm{j} .1529-8817.2001 .00102 . \mathrm{x}>$.

Bormans, M.; Ford, P. W.; Fabbro, L. Spatial and temporal variability in cyanobacterial populations controlled by physical processes. Journal of Plankton Research, v.27, n.1, p.61-70, 2005. <http://dx.doi.org/10.1093/plankt/fbh150>.

Bouvy, M.; Falcão, D.; Marinho, M.; Pagano, M.; Moura, A. Occurence of Cylindrospermopsis (Cyanobacteria) in 39 Brazilian tropical reservoirs during the 1998 drought. Aquatic Microbial Ecology, v.23, n.1, p.13-27, 2000. $<\mathrm{http}$ //dx.doi.org/10.3354/ame023013>.

Bouvy, M.; Molica, R.; Oliveira, S.; Marinho, M.; Becker, B. Effects of a cyanobacterial bloom (Cylindrospermopsis raciborskii) on bacteria and zooplankton communities in Ingazeira reservoir (northeast Brazil). Aquatic Microbial Ecology, v.25, n.3, p.215-227. 2001. <http://dx.doi. org/10.3354/ame025215>.

Bouvy, M.; Nascimento, S. M.; Molica, R. J. R.; Ferreira, A.; Huszar, V.; Azevedo, S. M. F. O. Limnological features in Tapacurá reservoir (northeast Brazil) during a severe drought. Hydrobiologia, v.493, n.1-3, p.115-130, 2003. $<$ http://dx.doi.org/10.1023/A:1025405817350>.

Carey, C. C.; Ibelings, B. W.; Hoffmann, E. P.; Hamilton, D. P.; Brookes, J. D. Eco-physiological adaptations that favour freshwater cyanobacteria in a changing climate. Water Research, v.46, n.6, p.1394-1407, 2012. <http://dx.doi. org/10.1016/j.watres.2011.12.016>.

Carvalho-De-La-Mora, L. M. Nostocophyceae (Cyanophyceae) de mananciais de abastecimento. I- Açude do Prata, Recife, Pernambuco. In: Encontro Brasileiro de Plâncton, 2., 1986, Salvador. Anais... Salvador: Universidade Federal da Bahia, 1986. p.7.

Chellappa, N. T.; Chellappa, S. L.; Chellappa, S. Harmful phytoplankton blooms and Fish mortality in a eutrophicated reservoir of northeast Brazil. Brazilian Archives of Biology and Technology, v.51, n.4, p.833-841, 2008. <http://dx.doi. org/10.1590/S1516-89132008000400022>.

Chorus, I.; Bartram, J. Toxic cyanobacteria in water. A guide to their public health consequences, monitoring and management. London: E \& FN Spon, 1999. 416p. <http:// dx.doi.org/10.4324/9780203478073>.

Costa, I. A. S.; Azevedo, S. M. F. O.; Senna, P. A. C.; Bernardo, R. R.; Costa, S. M.; Chellappa, N. T. Occurrence of toxin-producing cyanobacteria blooms in a Brazilian semiarid reservoir. Brazilian Journal of Biology, v.66, n.1b, p.211-219, 2006. <http://dx.doi.org/10.1590/S1519$69842006000200005>$. 
Costa, I. A. S.; Cunha, S. R. S.; Panosso, R.; Araújo, M. F. F.; Melo, J. L. S.; Eskinazi-Sant'Anna, E. M. Dinâmica de cianobactérias em reservatórios eutróficos do semi-árido do Rio Grande do Norte. Oecologia Brasiliensis, v.13, n.2, p.382-401, 2009. <http:/www. oecologiaaustralis.org/ojs/index.php/oa/article/view/ oeco.2009.1302.11/755>.

Cronberg, G.; Annadotter, H. Manual on aquatic cyanobacteria a photo and a synopsis of their toxicology. Copenhagen: Intergovernmental Oceanographic Commission of UNESCO; International Society for the Study of Harmful Algae, 2006. 106p.

Dantas, E. W.; Moura, A. N.; Bittencourt-Oliveira, M. C.; Neto, J. D. T. A.; Cavalcanti, A.D.C. Temporal variation of the phytoplankton community at short sampling intervals in the Mundaú reservoir, Northeastern Brazil. Acta Botanica Brasilica, v.22, n.4, p.970-982. 2008. <http://dx.doi.org/10.1590/S0102$33062008000400008>$.

Dantas, E. W.; Moura, A. N.; Bittencourt-Oliveira, M. C. Cyanobacterial blooms in stratified and destratified eutrophic reservoirs in semi-arid region of Brazil. Anais da Academia Brasileira de Ciências, v.83, n.4, p.1327-1338, 2011. <http://dx.doi.org/10.1590/S000137652011000400019>.

Domingos, P.; Rubim, T. K.; Molica, R. J. R.; Azevedo, S. M. F. O.; Carmichael, W. W. First report of microcystin production by picoplanktonic cyanobacteria isolated from a northeast Brazilian drinking water supply. Environmental Toxicology, v.14, n.1, p.31-35, 1999. <http://dx.doi. org/10.1002/(SICI)1522-7278(199902)14:1<31::AIDTOX6>3.0.CO;2-B $>$.

Domitrovic, Y. Z.; Forastier, M. E. Biodiversidad de Cyanophyceae (Cyanobacteria) y especies toxigénicas del litoral fluvial argentino. In: Aceñolaza, F. G. (Ed.). Temas de la Biodiversidad del Litoral Fluvial Argentino II. San Miguel de Tucumán: Insugeo, 2005. p.213-228. (Série Miscelânea, 14). <http://www.insugeo.org.ar/libros/ misc_14/pdf/17.pdf>. 19 Nov. 2012.

Huszar, V. L. M.; Silva, L. H. S.; Marinho, M.; Domingos, P.; Sant'anna, C. L. Cyanoprokaryote assemblages in eight produtive tropical Brazilian waters. Hydrobiologia, v.424, n.1-3, p.67-77, 2000. <http://dx.doi. org/10.1023/A:1003996710416>.

Jochimsen, E. M.; Carmichael, W. W.; An, J.; Cardo, D. M.; Cookson, S. T.; Holmes, C. E. M.; Antunes, B. C.; Melo Filho, D. A.; Lyra, T. M.; Barreto, V. S. T.; Azevedo, S. M. F. O.; Jarvis, W. R. Liver failure and death after exposure to microcystins at a hemodialysis center in Brazil. The New England Journal of Medicine, v.338, n.13, p.873-878, 1998. <http://dx.doi.org/10.1056/ NEJM199803263381304>.

Komárek, J. Planktic oscillatorialean cyanoprokariotes (short review according to combined phenotype and molecular aspects). Hydrobiologia, v.502, n.13, p.367-382, 2003. <http://dx.doi.org/10.1023/ B:HYDR.0000004294.17755.fe $>$.
Komárek, J.; Anagnostidis, K. Cyanoprokaryota. 2. Teil/Part 2: Oscillatoriales In: Büdel, B., Gärtner, G.; Krienitz, L.; Schargerl, M. (Hrsg.). Süßwasserflora von Mitteleuropa Bd 19/2: Cyanoprokaryota München: Elsevier GmbH, 2005. 759p. (Süßwasserflora von Mitteleuropa. Freshwater Flora of Central Europe, 19/2).

Komárek, J.; Anagnostidis, K. Modern approach to the classification system of Cyanophytes, 2: Chroococcales. Algological Studies/Archiv für Hydrobiologie, Supplement Volumes, n.43, p.157-226, 1986. <http://www. schweizerbart.de/papers/algol_stud/detail/43/63802>. 19 Nov. 2012.

Komárek, J.; Zapomělová, E. Planktic morphospecies of the cyanobacterial genus Anabaena = subg. Dolichospermum 1. part: coiled types. Fottea, v.7, n.1, p.1-31. 2007. <http:// fottea.czechphycology.cz/_contents/F07-2007-01.pdf $>$. 19 Nov. 2012.

Lira, G. A. S. T.; Araújo, E. L.; Bittencourt-Oliveira, M. C.; Moura, A. N. Phytoplankton abundance, dominance and coexistence in a eutrophic reservoir in the state of Pernambuco, Northeast Brazil. Anais da Academia Brasileira de Ciências, v.83, n.4, p.13131326. 2011. < http://dx.doi.org/10.1590/S000137652011000400018>.

Lira, G. A. S. T.; Moura, A. N.; Bittencourt-Oliveira, M. C.; Araújo, E. L. Comunidade fitoplanctônica e aspectos ecológicos de dois reservatórios eutróficos do nordeste do Brasil. In: Moura, A. N.; Araújo, E. L.; BittencourtOliveira, M. C.; Pimentel, R. M. M. P.; Albuquerque, U. P. (Eds.). Reservatórios do Nordeste do Brasil: Biodiversidade, ecologia e manejo. 1.ed. Nuppea: Recife, 2010. p.145-168.

Litchman, E.; Tezanos Pinto, P.; Klausmeier, C. A.; Thomas, M. K.; Yoshiyama, K. Linking traits to species diversity and community structure in phytoplankton. Hydrobiologia, v.653, n.1, p.15-28, 2010. <http://dx.doi.org/10.1007/ s10750-010-0341-5>.

Mateucci, S. D.; Colma, A. La metodologia para el estudo da vegetación. Washington: OEA, 1982. p.168. (Série Biologia. Monografías Científicas, 22). < http://aprobioma. files.wordpress.com/2011/03/metod_para_el_estudio_de la_vegetacion_archivo1.pdf $>$. 19 Nov. 2012.

Moura, A. N.; Araújo, M. K. C.; Soriano, H. B. O.; Lira, G. A. S. T.; Nascimento, E. C. Cianobactérias planctônicas em reservatório eutrófico do estado de Pernambuco. In: Moura, A. N.; Araújo, E. L.; Bittencourt-Oliveira, M. C.; Pimentel, R. M. M. P.; Albuquerque, U. P. (eds.). Reservatórios do Nordeste do Brasil: Biodiversidade, ecologia e manejo. 1.ed. Nuppea: Recife, 2010. p.115-143.

Moura, A. N.; Dantas, E. W.; Bittencourt-Oliveira, M. C. Struture of the phytoplankton in a water supply system in the state o Pernambuco- Brazil. Brazilian Archives of Biology and Technology, v.50, n.4, p.645-654, 2007. <http://dx.doi.org/10.1590/S1516$89132007000400010>$. 
Moura,A.N.;Dantas,E.W.; Oliveira,H.S.B.;Bittencourt-Oliveira, M. C. Vertical and temporal dynamics of cyanobacteria in the Carpina potable water reservoir in northeastern Brazil. Brazilian Journal of Biology, v.71, n.2, p.1-9, 2011. <http:// dx.doi.org/10.1590/S1519-69842011000300015>.

Paerl, H. W.; Huisman, J. Blooms like it hot. Science, v.320, n.5872, p.57-58, 2008. <http://dx.doi.org/10.1126/ science.1155398>.

Panosso, R.; Costa, I. A. S.; Souza, N. R.; Attayde, J. L.; Cunha, S. R. S.; Gomes, F. C. F. Cianobactérias e cianotoxinas em reservatórios do estado do Rio Grande do Norte e o potencial controle de florações pela tilápia do Nilo (Oreochromis niloticus). Oecologia brasiliensis, v.11, n.3, p.433-449, 2007. $<\mathrm{http} / / /$ dx.doi.org/10.1590/S1516-89132007000400010>.
Wacklin, P.; Hoffmann, L.; Komárek, J. Nomenclatural validation of the genetically revised cyanobacterial genus Dolichospermum (Ralfs ex Bornet et Flahault) comb. nova. Fottea, v.9, n.1, p.59-64, 2009. <http:// fottea.czechphycology.cz/_contents/F09-1-200905.pdf $>$.

Zapomělová, E.; Jezberová, J.; Hrouzek, P.; Hisem, D.; Reháková, K.; Komárková. J. Polyphasic characterization of three strains of Anabaena reniformis and Aphanizomenon aphanizomenoides (Cyanobacteria) and their reclassification to Sphaerospermum Gen. Nov. (incl. Anabaena kisseleviana). Nomenclatural Note. Journal of Phycology, v.46, n.2, p.415, 2010. <http://dx.doi.org/10.1 $111 / \mathrm{j} .1529-8817.2010 .00830 . \mathrm{x}>$. 